\title{
Sistema Integrado de Diagnóstico y Recomendación (DRIS) para piña (Ananas comosus), variedad Oro Miel (MD-2)
}

\section{Diagnosis and Recommendation Integrated System (DRIS) for pineapple (Ananas comosus), Honey Gold variety (MD-2)}
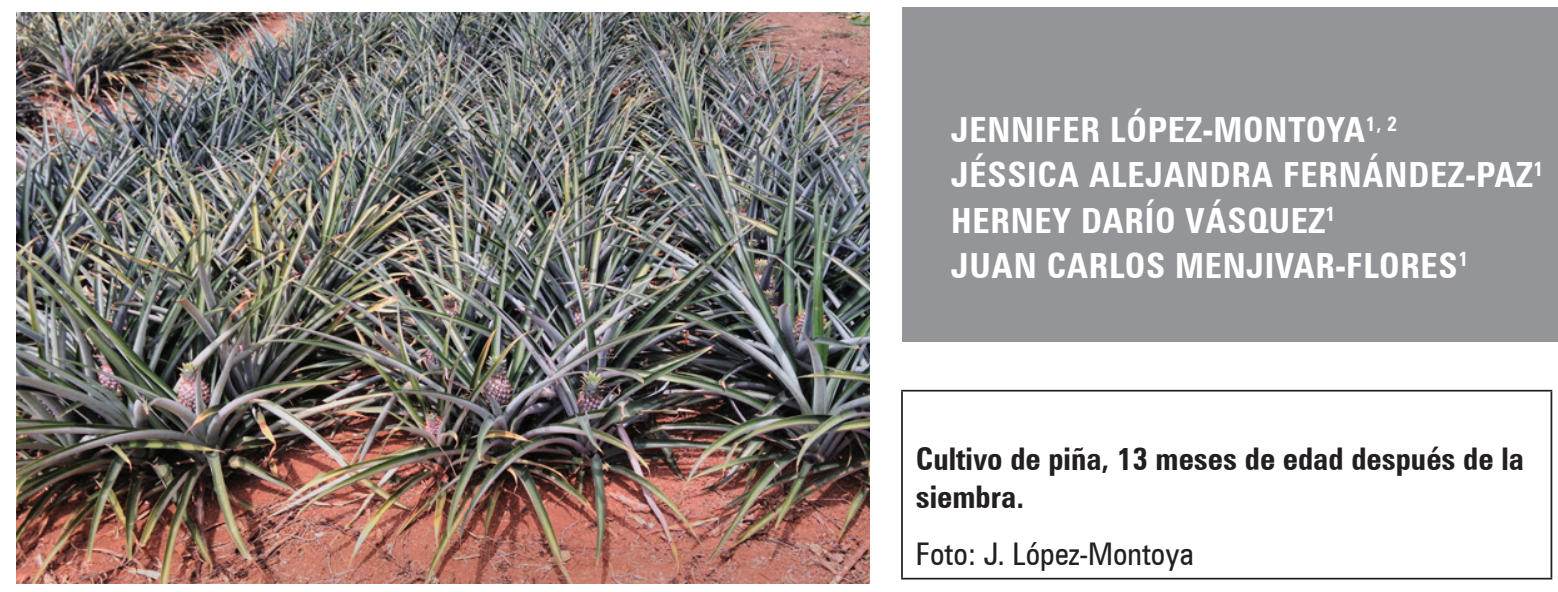

\section{RESUMEN}

La piña (Ananas comosus), es la tercera especie tropical más importante, se cultiva en países tropicales y subtropicales, en ese orden de ideas y dada la importancia del cultivo, se hace necesario contar con una herramienta para un adecuado diagnóstico nutricional, es así como la presente investigación buscó determinar las normas DRIS (Sistema Integrado de Diagnóstico y Recomendación) para el cultivo de piña, esta es una metodología de interpretación de análisis foliares, basada principalmente en el establecimiento de poblaciones de referencia para la obtención de normas o relaciones ideales de nutrientes, las cuales sirven para la interpretación del contenido nutricional de una determinada especie. Se utilizó un diseño en bloques completos al azar, con seis tratamientos y cuatro repeticiones basados en diferentes planes de fertilización muestreando cuatros etapas del cultivo. Se usó un suelo con $\mathrm{pH}$ de 3. Los resultados mostraron deficiencias de B, S y Zn, elementos como el $\mathrm{Cu}$ y $\mathrm{Na}$ presentaron limitaciones por excesos seguido del Fe solo para la primera etapa y el $\mathrm{P}$ fue uno de los elementos minerales que presentó un equilibrio durante toda la etapa del cultivo. El orden decreciente de las limitantes por deficiencia para concentraciones en tejido foliar en piña se dividió en cuatro etapas de desarrollo del cultivo, la primera etapa de 3 meses después de la siembra, se encontró $\mathrm{Mn}>\mathrm{B}>\mathrm{S}>\mathrm{Ca}>\mathrm{K}>\mathrm{Mg}>\mathrm{Cu}>\mathrm{N}>\mathrm{P}>\mathrm{Z}$ $\mathrm{n}>\mathrm{Na}>\mathrm{Fe}$, la segunda etapa de 6 meses después de la siembra $\mathrm{Zn}>\mathrm{B}>\mathrm{Na}>\mathrm{Ca}>\mathrm{Mn}>\mathrm{Mg}>\mathrm{K}>\mathrm{N}>\mathrm{P}>\mathrm{S}>\mathrm{Cu}$, la tercera etapa de 9 meses después de la siembra $\mathrm{B}>\mathrm{Zn}>\mathrm{S}>\mathrm{Mn}>\mathrm{Ca}>\mathrm{N}>\mathrm{K}>\mathrm{P}>\mathrm{Mg}>\mathrm{Na}>\mathrm{Cu}$, y la última etapa de 12 meses después de la siembra $\mathrm{B}>\mathrm{Zn}>\mathrm{Mn}>\mathrm{Ca}>\mathrm{Mg}>\mathrm{N}>\mathrm{K}>\mathrm{S}>\mathrm{Na}>\mathrm{P}>\mathrm{Cu}$.

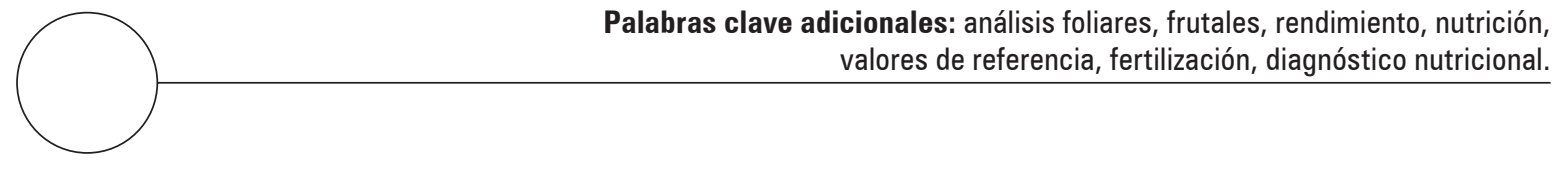

1 Facultad de Ciencias Agropecuarias, Universidad Nacional de Colombia, Palmira (Colombia). ORCID López-Montoya, J.: 0000-0003-2565-5436; ORCID Fernández-Paz, J.A.: 0000-0002-1307-4055; ORCID Vásquez, H.D.: 0000-0001-9579-5149; ORCID Menjivar-Flores, J.C.: 0000-0002-0985-7778

2 Autor para correspondencia. jelopezmo@unal.edu.co 


\section{ABSTRACT}

Pineapple (Ananas comosus) is the third most important tropical species and is grown in tropical and subtropical countries. Given the importance of this crop, it is necessary to have a tool for proper nutritional diagnosis. So, the present study sought to determine the DRIS (Integrated Diagnostic and Recommendation System) standards for the cultivation of pineapple, that is, a methodology for the interpretation of foliar analyzes, based mainly on the establishment of reference populations for obtaining norms or ideal relationships of nutrients, which serve for the interpretation of the nutritional content of a certain species. A randomized complete block design was used, with six treatments and four repetitions, based on different fertilization plans, sampling four stages of the crop. A soil with a pH of 3 was used. The results showed deficiencies of $\mathrm{B}, \mathrm{S}$ and $\mathrm{Zn}$; elements such as $\mathrm{Cu}$ and $\mathrm{Na}$ showed limitations as a result of excesses, followed by Fe, but only for the first stage, while $\mathrm{P}$ was one of the mineral elements that presented a good balance during the entire stage of the crop. The decreasing order of deficiency constraints for the leaf tissue concentrations in the pineapple was divided into four stages of crop development: the first stage 3 months after sowing: $\mathrm{Mn}>\mathrm{B}>\mathrm{S}>\mathrm{Ca}>\mathrm{K}>\mathrm{Mg}>\mathrm{Cu}>\mathrm{N}>\mathrm{P}>\mathrm{Zn}>\mathrm{Na}>\mathrm{Fe}$, the second stage 6 months after sowing: $\mathrm{Zn}>\mathrm{B}>-$ $\mathrm{Na}>\mathrm{Ca}>\mathrm{Mn}>\mathrm{Mg}>\mathrm{K}>\mathrm{N}>\mathrm{P}>\mathrm{S}>\mathrm{Cu}$, the third stage 9 months after sowing: $\mathrm{B}>\mathrm{Zn}>\mathrm{S}>\mathrm{Mn}>\mathrm{Ca}>\mathrm{N}>\mathrm{K}>\mathrm{P}>\mathrm{M}$ $\mathrm{g}>\mathrm{Na}>\mathrm{Cu}$, and the last stage 12 months after sowing: $\mathrm{B}>\mathrm{Zn}>\mathrm{Mn}>\mathrm{Ca}>\mathrm{Mg}>\mathrm{N}>\mathrm{K}>\mathrm{S}>\mathrm{Na}>\mathrm{P}>\mathrm{Cu}$.

Additional key words: foliar analysis, interpretation, nutrients, fruit plants, yield, nutrition, reference values, fertilization, nutritional diagnosis.

Fecha de recepción: 16-10-2017 Aprobado para publicación: 30-05-2018

INTRODUCCIÓN

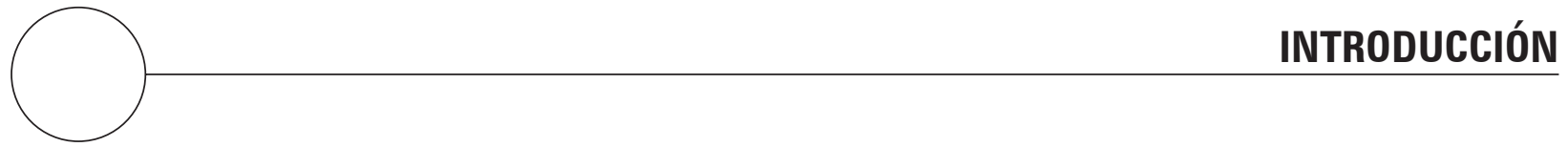

Ananas comosus (L.) Merr., es una planta frutícola herbácea perenne tropical perteneciente a la familia Bromeliaceae, su centro de origen está ubicado en América del Sur en la región del Mattogroso entre Uruguay y Brasil (León, 2000) y se conoce con los nombres de piña, naná, ananás, piña tropical y piña de azúcar (García y Serrano, 2005).

A nivel mundial la piña ocupa el tercer lugar entre las frutas tropicales, después del banano y los cítricos (Sema et al., 2010). Los cinco mayores productores son los países de Tailandia, Brazil, Indonesia, Filipinas y Costa Rica, mientras el 70\% de su producón es consumida internamente, solamente el $30 \%$ es exportado (Hassan et al., 2011).

En Colombia, para el año 2016, se produjeron 801.081 t, de un total de 18.378 ha cosechadas y los departamentos como Santander, Meta, Valle del Cauca y Cauca ocupan los primeros lugares en producción (Ministerio de Agricultura, 2016). Combatt et al. (2008) reportan que la piña se cultiva en diferentes suelos en Colombia y solamente en pocos casos se aplican las correcciones necesarias.

"El manejo de la fertilización es uno de los factores de mayor relevancia en el cultivo de piña" (Marca-Huamancha et al., 2018). Actualmente existen métodos eficientes para el diagnóstico nutricional de los cultivos, entre los que se encuentran de uso frecuente son el diagnóstico de los niveles críticos, rangos de suficiencia y el Sistema Integrado de Diagnóstico y Recomendación (DRIS), solo por mencionar algunos (Chacón, 2012). El DRIS, por sus siglas en inglés, "es un conjunto de normas de diagnóstico nutricional de cultivos, mediante la calibración de la composición de los tejidos, la composición del suelo, las condiciones ambientales y prácticas integrales de manejo del cultivo" (Rodríguez y Rodríguez, 2000).

El DRIS fue propuesto inicialmente por Beaufils en la década de los años 60's (Sumner, 1977), en proyectos de fisiología y nutrición vegetal para el cultivo de caucho en Vietnam, maíz y caña de azúcar en Suráfrica (Rodríguez y Rodríguez, 2000). Muchos países cuentan con estas normas para diversos cultivos, existen reportes de investigaciones en café (Da Costa et al., 1997), caña (Beaufils y Sumner, 1976; Elwali y Gascho, 1984), maíz (Baldock y Schulte, 1996), limón (Cerda et al., 1995), piña (Angeles et al., 1990), vid (Sharma et al., 2005), soya (Bell et al., 1995), entre otros.

Para el establecimiento de la norma es necesario contar con poblaciones de referencia basadas en valores 
de alto y bajo rendimiento para el cultivo de estudio (Chacón, 2012). A partir de los datos de rendimiento y análisis foliares, se originan relaciones directas o inversas entre pares de nutrientes (Urricariet et al., 2004) para obtener los índices que finalmente son interpretados en la tabla de referencia propuesta por Wadt (1996).

Es de resaltar que el cultivo de la piña ha sido bien aceptado a nivel mundial, pues atrae a productores-inversionistas. Es una planta de metabolismo CAM (Crassula acid metabolism) con eficiente uso de agua (Vásquez et al., 2012). A pesar de ello, hay una serie de factores de gran relevancia que limitan la producción y óptima calidad, como es la nutrición vegetal (Herrera, 2015). En Colombia las investigaciones en normas DRIS son escasas para varios cultivos. La interpretación de los análisis foliares se realiza a través del nivel crítico o rangos de suficiencia en la mayoría de los casos, en ese sentido el cultivo de piña carece de investigación local con resultados acordes a las condiciones del país, ya que el diagnóstico nutricional se realiza con datos de otros países.

El objetivo de este trabajo fue desarrollar el Sistema Integrado de Diagnóstico y Recomendación (DRIS), para el cultivo de piña (Ananas comosus), variedad oro miel (MD-2), en condiciones edafoclimaticas del municipio de Santander de Quilichao, Cauca (Colombia).

\section{MATERIALES Y MÉTODOS}

La investigación se realizó en el municipio de Santander de Quilichao, Cauca (Colombia), a 03 30'53,2" $\mathrm{N}$ y 76¹8'02,2' W, una altitud de $1.030 \mathrm{msnm}$, con una temperatura media de $27^{\circ} \mathrm{C}$, humedad relativa del $60 \%$ y una precipitación promedia de $1.800 \mathrm{~mm}$ año-1 (Ideam, 2016). Según análisis, el tipo de suelo fue franco-arcilloso, con un $\mathrm{pH}$ de 3,6, muy por debajo de lo recomendado por Vásquez et al. (2012) con un óptimo entre 5 y 6.

Se evaluó plantas de piña variedad MD-2, establecidas a una densidad de siembra de 66.000 plantas/ha. Las unidades experimentales (UE) fueron divididas en parcelas de $4,5 \times 5 \mathrm{~m}$ para un área de $22,5 \mathrm{~m}^{2}$, seis parcelas por cada bloque, con una separación de parcelas de $1 \mathrm{~m}^{2}$ y la separación entre bloques de $2 \mathrm{~m}^{2}$; el área total tenía $50 \mathrm{~m}^{2}$ largo por $20 \mathrm{~m}^{2}$ ancho.

Se utilizó un diseño en bloques completos al azar con seis tratamientos y cuatro repeticiones, para un total de 24 unidades experimentales. Los tratamientos consistieron en la aplicación de diferentes niveles de NPK teniendo en cuenta las concentraciones de los nutrientes en el suelo y los planes de fertilización que aplican los agricultores de la zona. La concentración de nutrientes en el suelo sin fertilizar se tomó como tratamiento testigo T0; T1 y T2 fueron tratamientos recomendados por agricultores de la zona, los tratamientos T3, T4, T5 fueron tratamientos propuestos por los investigadores guardando relación entre los nutrientes NPK y la concentración de estos en el suelo según el resultado del análisis de suelos, los demás elementos se aplicaron de acuerdo con las dosis establecidas por los agricultores. En la tabla 1 se muestran la descripción de los tratamientos, dosis y productos aplicados.

Se colectaron en horas de la mañana un total de 72 muestras foliares de acuerdo con la metodología planteada por Centro Internacional para Agricultura Tropical (CIAT, 1993), consistió en colectar la planta completa y se tomaron hojas de la parte baja, media y alta de la planta; en cuatro etapas de desarrollo del

Tabla 1. Descripción de tratamientos, dosis y productos evaluados en piña 'Oro Miel' en Santander de Quilichao, Colombia.

\begin{tabular}{|c|c|c|c|c|c|c|}
\cline { 2 - 7 } \multicolumn{1}{c|}{} & \multicolumn{6}{c|}{ Fertilizante aplicado por planta (g/unidad experimental) } \\
\hline Tratamiento & $\begin{array}{c}\text { Urea } \\
{[\mathrm{CO}(\mathrm{NH})]}\end{array}$ & $\begin{array}{c}\text { Fosfato diamónico } \\
{\left[\left(\mathrm{NH}_{42} \mathrm{HPO}_{4}\right]\right.}\end{array}$ & $\begin{array}{c}\text { Cloruro de potasio } \\
{[\mathrm{KCl}]}\end{array}$ & $\begin{array}{c}\text { Sulfato de potasio } \\
{\left[\mathrm{K}_{2} \mathrm{SO}_{4}\right]}\end{array}$ & $\begin{array}{c}\text { Sulfato de amonio } \\
{\left[\left(\mathrm{NH}_{4}\right)_{2} \mathrm{SO}_{4}\right]}\end{array}$ & $\begin{array}{c}\text { Fosfato } \\
\text { monoamónico } \\
{\left[\mathrm{NH}_{4} \mathrm{H}_{2} \mathrm{PO}_{4}\right]}\end{array}$ \\
\hline T0 & 0 & 0 & 0 & 0 & 0 & 0 \\
\hline T1 & 8.125 & 2.250 & 6.000 & 4.750 & 750 & 0 \\
\hline T2 & 13.000 & 1.750 & 8.125 & 4.500 & 0 & 0 \\
\hline T3 & 5.068 & 4.656 & 4.792 & 4.500 & 0 & 0 \\
\hline T4 & 6.464 & 4.884 & 4.872 & 4.500 & 0 & 0 \\
\hline T5 & 7.528 & 7.444 & 6.264 & 4.500 & & 0 \\
\hline
\end{tabular}


cultivo 3, 6, 9 meses en etapa vegetativa y a los 12 meses etapa de floración en cada una de las etapas se tomaron un número total de 18 muestras, tres repeticiones por tratamiento. Las variables de respuesta fueron el contenido de nutrientes en $\mathrm{g} \mathrm{kg}^{-1}$ de peso seco y rendimiento en $\mathrm{kg}$. Se utilizó un análisis de varianza para comparar las dos poblaciones (de referencia y no referencia).

Las muestras fueron llevadas al laboratorio de servicios analíticos del CIAT para su análisis químico, en el cual se determinó $\mathrm{N}$ y $\mathrm{P}$ por espectrometría, $\mathrm{Ca}, \mathrm{Mg}$, $\mathrm{Cu}, \mathrm{Zn}, \mathrm{Mn}$ y Fe total por absorción atómica (Ab. At), $\mathrm{Na}$ por Em. Atómica, B por espectrometría Azometina y $S$ total por turbidimentria para las muestras foliares. El laboratorio contaba con una temperatura de $22 \pm 3^{\circ} \mathrm{C}$ y humedad relativa $60 \pm 5 \%$.

Para determinar las poblaciones de referencia (alto y bajo rendimiento), se partió de los análisis foliares y los valores de rendimiento; estos se calcularon a partir de la multiplicación del peso fresco promedio del fruto por la densidad de plantas establecida en el lote experimental, según lo propuesto por Rebolledo et al. (2006), fueron organizados en una hoja de cálculo de Microsoft Excel, a estos se les realizó un análisis estadístico para la obtención de medias, desviaciones estándar y coeficientes de variación (Herrera, 2015). Con los resultados anteriores se obtuvo la media general de todos los datos de rendimiento y aquellos valores que estuvieron por encima de la media general se escogieron como población de referencia y los que estuvieran por debajo de la media general, fueron la población de no referencia.

\section{Obtención de los índices DRIS foliares e interpretación}

Las normas del Sistema Integrado de Diagnóstico y Recomendación, se determinaron a partir de la metodología propuesta por Sumner (1977), en donde las relaciones directas e inversas entre los elementos, dos a dos para la población de alto y bajo rendimiento, expresando que "la relación es directa si el nutriente en cuestión aparece en el numerador (A/B), y la relación es inversa si aparece en el denominador" (Mourão-Filho, 2004), además de ello se calculó para cada una de las relaciones, la media (x), la desviación estándar (s), la varianza (S) y el coeficiente de variación $(\mathrm{CV})$. Y para saber el número de relaciones posibles (RP) se utilizó la fórmula (1) reportada por Herrera (2015).

$$
\mathrm{RP}=n(n-1)
$$

donde, $n$ es el número de nutrientes en estudio.

Para la obtención de las relaciones que se convirtieron en norma o relaciones ideales, se usó la metodología utilizada por Beaufils (1976), en donde se tiene en cuenta las varianzas de las distintas relaciones en cada una de las poblaciones (referencia y no referencia), seleccionando las relaciones que presentan menor variabilidad (Franco, 2007). Para ello se dividió las varianzas de la población de no referencia sobre la varianza de la población de referencia (Chacón, 2012).

Para cada una de las muestras tomadas, se calcularon los índices DRIS, utilizando como valores de referencia el promedio de las concentraciones en la población de bajo rendimiento, las funciones DRIS se calculan con el método de Beaufils (Chacón, 2012). "Esto con el fin de identificar los posibles desbalances nutricionales y establecer el orden de requerimiento para cada uno de los nutrientes a nivel foliar" (Sánchez et al., 2009).

$\int \frac{A}{B}=\frac{A / B}{a / b}-1 \frac{1000}{C V}$
2) $\int \frac{A}{B}=1-\frac{a / b}{A / B} \frac{1000}{C V}$

donde, $A / B$ es relación de la muestra, $a / b$ relación de población de referencia, $C V$ coeficiente de variación. Se aplicó el caso 1) cuando $A / B$ es mayor o igual a $a / b$, fórmula (2) y el caso 2) cuando $A / B$ es menor o igual a $a / b$, fórmula (3).

Una vez realizadas las funciones de cada una de las relaciones, se calcularon los índices de los elementos involucrados y se determinó las ecuaciones generales de los índices DRIS para cada uno de los elementos según García (2000).

Cada uno de los índices es la medida de las funciones de las relaciones donde está el nutrimento. "Si la relación es directa se le respeta el signo (+), pero si es inversa se le cambia de signo (-)". Una vez obtenido el cálculo de cada uno de los índices de los nutrientes, se establece el Índice de Balance Nutricional (IBN), el cual es una suma algebraica de los valores absolutos de cada uno de los índices para cada elemento mineral (Chacón, 2012), así: 
$\mathrm{IBN}=|\mathrm{I}(\mathrm{N})|+|\mathrm{I} \quad(\mathrm{P})|+|\mathrm{I} \quad(\mathrm{K})|+|\mathrm{I} \quad(\mathrm{Ca})|+\mid \mathrm{I}$ $(\mathrm{Mg})|+| \mathrm{I} \quad(\mathrm{Na})|+| \mathrm{I} \quad(\mathrm{Cu})|+| \mathrm{I} \quad(\mathrm{Zn})|+| \mathrm{I}$ $(\mathrm{Mn})|+| \mathrm{I}(\mathrm{Fe})|+| \mathrm{I}(\mathrm{S})|+| \mathrm{I}(\mathrm{B}) \mid$

También se calculó el IBN medio (Wadt, 1999), el cual se determina mediante el resultado de IBN sobre el número de índices (n), así.

$$
\mathrm{IBNm}=\mathrm{IBN} / \mathrm{n}
$$

\section{Interpretación de los índices DRIS}

Wadt (1996) clasificó los valores obtenidos para cada nutriente de acuerdo con el potencial de respuesta al manejo de la fertilización realizado (Tab. 2).

Tabla 2. Criterio para la interpretación de los índices DRIS.

\begin{tabular}{|c|c|}
\hline Criterio & Estado nutricional \\
\hline $\begin{array}{c}\mid(N)<0 y \\
|I(N)|>I B N m\end{array}$ & Deficiente \\
\hline $\begin{array}{c}\mid(N)>0 y \\
|I(N)| \leq \mid B N m\end{array}$ & Equilibrado \\
\hline$|\mathrm{I}(\mathrm{N})|>\mathrm{IBNm}$ & Exceso \\
\hline
\end{tabular}

N: corresponde al nutriente de análisis. Fuente: adaptado de Wadt (1996).

\section{RESULTADOS Y DISCUSIÓN}

Se estableció la población de referencia basados principalmente en el promedio general del rendimiento de los tratamientos (Tab. 3).

Tabla 3. Promedios de rendimiento (por unidad experimental) de piña 'Oro miel' en los tratamientos evaluados para Santander de Quilichao, Colombia.

\begin{tabular}{|c|c|}
\hline \multicolumn{2}{|c|}{ Valores promedio de rendimiento $(\mathrm{kg})$} \\
\hline T0 (Testigo) & 446,75 \\
\hline T1 & 939,25 \\
\hline T2 & 742,00 \\
\hline T3 & 786,50 \\
\hline T4 & 743,00 \\
\hline T5 & 946,00 \\
\hline Promedio & 767,25 \\
\hline
\end{tabular}

Tratamientos descritos en tabla 1.

Esta selección cumple con los criterios establecidos por Letzsch y Sumner (1984), quienes postularon que la población de referencia al menos debe contener un
$10 \%$ de las observaciones de una base de datos global, esto con el fin de garantizar diferencias significativas con la población de no referencia. Para población de referencia se tuvo un rendimiento total de 2.671,75 $\mathrm{kg} / \mathrm{unidad}$ experimental, comparado con la población de baja producción que solo obtuvo un rendimiento de $1.931,75 \mathrm{~kg} /$ unidad experimental.

Hay que tener en cuenta que para las 132 relaciones posibles (RP) de los nutrientes evaluados en los análisis foliares, se debe determinar las cuatro etapas y las dos poblaciones. Se calculó para cada relación: media, desviación estándar, varianza y coeficiente de variación para la obtención de las normas (Urricariet et al., 2004). La tabla 4 presenta los valores obtenidos de los nutrientes evaluados en cada uno de los análisis foliares.

En consecuencia de lo anterior, se puede decir que la varianza por lo general es menor en una población de referencia que en una población de baja producción (Herrera, 2015). También que el valor promedio de $\mathrm{N}$ en las dos primeras etapas de desarrollo del cultivo es mayor en la población de alto rendimiento, contrario a lo que sucede en las dos siguientes etapas que el contenido promedio disminuye conforme avanza la edad de la planta (Chacón, 2012).

Por otra parte, en la población de no referencia el contenido promedio de $\mathrm{N}$ se mantiene casi estable durante toda la etapa de crecimiento, esto puede deberse a que las plantas de la población de alta productividad aprovecharon el $\mathrm{N}$ en las primeras etapas de desarrollo para la formación de estructuras como tejidos o alimento para la planta (Marschner, 2011), pero que en la población de no referencia la planta se mantuvo en un tamaño y peso constante y no necesito del $\mathrm{N}$ esencialmente para la formación de algún tipo de estructura la cual le permitiera tener mejor rendimiento. El $\mathrm{K}$ que es uno de los elementos que aumentó considerablemente en todas las etapas de desarrollo en las dos poblaciones.

El P en la población de referencia presenta una mayor absorción en la segunda etapa del cultivo y en las dos últimas etapas esta absorción disminuye, este comportamiento es similar al de la población de no referencia, pero esta población presenta menores cantidades de absorción.

El K presenta un comportamiento y absorción parecido en las dos poblaciones, incrementan la demanda de $\mathrm{K}$ a medida que avanza el crecimiento del cultivo, 
Tabla 4. Análisis foliares de piña 'Oro miel' en diferentes etapas de crecimiento para la población de referencia y no referencia de un estudio DRIS.

\begin{tabular}{|c|c|c|c|c|c|c|c|c|}
\hline \multirow{2}{*}{ Variable } & \multicolumn{4}{|c|}{ Población de referencia } & \multicolumn{4}{|c|}{ Población de no referencia } \\
\hline & Media & Desviación & Varianza & Coeficiente & Promedio & Desviación & Varianza & Coeficiente \\
\hline \multicolumn{9}{|c|}{ Etapa 1 (3 meses de edad) } \\
\hline$N\left(g ~ k g^{-1}\right)$ & 18,1 & 0,53 & 0,28 & 11,21 & 17,79 & 4,93 & 24,32 & 0,27 \\
\hline$P\left(g_{k g}{ }^{-1}\right)$ & 1,26 & 0,17 & 0,03 & 0,13 & 1,14 & 0,29 & 1,21 & 0,25 \\
\hline $\mathrm{K}\left(\mathrm{g} \mathrm{kg}^{-1}\right)$ & 10,46 & 1,80 & 3,25 & 0,17 & 12,79 & 4,58 & 20,99 & 0,35 \\
\hline Ca $\left(\mathrm{g} \mathrm{kg}^{-1}\right)$ & 2,46 & 0,42 & 0,18 & 0,17 & 2,82 & 0,70 & 0,50 & 0,24 \\
\hline $\operatorname{Mg}\left(g \mathrm{~kg}^{-1}\right)$ & 1,98 & 0,18 & 0,03 & 0,09 & 2,12 & 0,48 & 0,23 & 0,22 \\
\hline $\mathrm{Na}\left(\mathrm{g} \mathrm{kg}^{-1}\right)$ & 0,073 & 0,0040 & 0,00002 & 0,05 & 0,07 & 0,02 & 0,00 & 0,27 \\
\hline $\mathrm{Cu}\left(\mathrm{g} \mathrm{kg}^{-1}\right)$ & 0,02 & 0,013 & 0,00017 & 0,56 & 0,03 & 0,02 & 0,00 & 0,56 \\
\hline $\mathrm{Zn}\left(\mathrm{g} \mathrm{kg}^{-1}\right)$ & 0,070 & 0,011 & 0,00012 & 0,15 & 0,06 & 0,02 & 0,00 & 0,34 \\
\hline $\operatorname{Mn}\left(\mathrm{g} \mathrm{kg}^{-1}\right)$ & 0,24 & 0,05 & 0,0025 & 0,2 & 0,38 & 0,18 & 0,03 & 0,46 \\
\hline $\mathrm{Fe}\left(\mathrm{g} \mathrm{kg}^{-1}\right)$ & 0,34 & 0,07 & 0,004 & 0,2 & 0,36 & 0,07 & 0,01 & 0,2 \\
\hline$S\left(g_{k g}^{-1}\right)$ & 1,49 & 0,16 & 0,03 & 0,1 & 1,60 & 0,33 & 0,11 & 0,2 \\
\hline$B\left(\mathrm{~g} \mathrm{~kg}^{-1}\right)$ & 0,0093 & 0,0004 & 0 & 0,043 & 0,01 & 0,001 & 0,00 & 0,01 \\
\hline \multicolumn{9}{|c|}{ Etapa 2 (6 meses de edad) } \\
\hline $\mathrm{N}\left(\mathrm{g} \mathrm{kg}^{-1}\right)$ & 23,21 & 1,92 & 3,68 & 0,08 & 19,78 & 3,95 & 15,60 & 0,19 \\
\hline$P\left(g ~ k g^{-1}\right)$ & 1,50 & 0,11 & 0,01 & 0,06 & 1,40 & 0,12 & 0,01 & 0,08 \\
\hline$K\left(g_{k g}{ }^{-1}\right)$ & 37,67 & 5,85 & 34,23 & 0,15 & 40,41 & 3,05 & 9,30 & 0,075 \\
\hline Ca $\left(\mathrm{g} \mathrm{kg}^{-1}\right)$ & 1,48 & 0,32 & 0,10 & 0,21 & 2,53 & 1,00 & 0,99 & 0,39 \\
\hline $\operatorname{Mg}\left(\mathrm{kg}^{-1}\right)$ & 1,72 & 0,33 & 0,11 & 0,19 & 2,14 & 0,62 & 0,38 & 0,28 \\
\hline $\mathrm{Na}\left(\mathrm{g} \mathrm{kg}^{-1}\right)$ & 0,10 & 0,03 & 0,001 & 0,3 & 0,09 & 0,02 & 0,001 & 0,25 \\
\hline $\mathrm{Cu}\left(\mathrm{g} \mathrm{kg}^{-1}\right)$ & 0,03 & 0,01 & 0,0001 & 0,33 & 0,03 & 0,01 & 0,0001 & 0,36 \\
\hline Zn $\left(\mathrm{g} \mathrm{kg}^{-1}\right)$ & 0,08 & 0,01 & 0,0001 & 0,12 & 0,04 & 0,01 & 0,0002 & 0,35 \\
\hline $\operatorname{Mn}\left(\mathrm{g} \mathrm{kg}^{-1}\right)$ & 0,31 & 0,03 & 0,001 & 0,1 & 0,41 & 0,11 & 0,01 & 0,26 \\
\hline $\mathrm{Fe}\left(\mathrm{g} \mathrm{kg}^{-1}\right)$ & 0,0003 & 0,0001 & 0 & & 0,00 & 0,0001 & 0 & \\
\hline$S\left(g_{k g}^{-1}\right)$ & 1,74 & 0,08 & 0,01 & 0,045 & 1,67 & 0,21 & 0,04 & 0,12 \\
\hline$B\left(g_{k g}^{-1}\right)$ & 0,04 & 0,03 & 0,00 & 0,75 & 0,02 & 0,01 & 0,0001 & 0,4 \\
\hline \multicolumn{9}{|c|}{ Etapa 3 (9 meses de edad) } \\
\hline $\mathrm{N}\left(\mathrm{g} \mathrm{kg}^{-1}\right)$ & 18,54 & 3,02 & 9,10 & 0,16 & 19,03 & 3,12 & 9,74 & 0,16 \\
\hline$P\left(g ~ k g^{-1}\right)$ & 0,97 & 0,07 & 0,00 & 0,07 & 0,82 & 0,11 & 0,01 & 0,13 \\
\hline$K\left(\mathrm{~g} \mathrm{~kg}^{-1}\right)$ & 47,54 & 2,76 & 7,61 & 0,05 & 44,82 & 3,87 & 14,99 & 0,08 \\
\hline Ca $\left(\mathrm{g} \mathrm{kg}^{-1}\right)$ & 2,39 & 0,55 & 0,30 & 0,23 & 2,73 & 0,79 & 0,62 & 0,28 \\
\hline $\mathrm{Mg}\left(\mathrm{g} \mathrm{kg}^{-1}\right)$ & 2,09 & 0,39 & 0,15 & 0,18 & 2,33 & 0,47 & 0,23 & 0,2 \\
\hline $\mathrm{Na}\left(\mathrm{g} \mathrm{kg}^{-1}\right)$ & 0,06 & 0,01 & 0,0001 & 0,16 & 0,06 & 0,01 & 0,0001 & 0,16 \\
\hline $\mathrm{Cu}\left(\mathrm{g} \mathrm{kg}^{-1}\right)$ & 0,02 & 0,01 & 0,0001 & 0,5 & 0,02 & 0,01 & 0,0001 & \\
\hline $\mathrm{Zn}\left(\mathrm{g} \mathrm{kg}^{-1}\right)$ & 0,04 & 0,004 & 0 & & 0,03 & 0,01 & 0,0001 & 0,33 \\
\hline $\operatorname{Mn}\left(\mathrm{g} \mathrm{kg}^{-1}\right)$ & 0,24 & 0,04 & 0,0017 & 0,16 & 0,29 & 0,06 & 0,0038 & 0,2 \\
\hline $\mathrm{Fe}\left(\mathrm{g} \mathrm{kg}^{-1}\right)$ & 0,0002 & 0,00003 & 0 & & 0,0001 & 0,00002 & 0,0000 & \\
\hline$S\left(g_{k g}^{-1}\right)$ & 2,19 & 0,17 & 0,03 & 0,07 & 2,23 & 0,22 & 0,05 & 0,09 \\
\hline$B\left(g_{k g}^{-1}\right)$ & 0,03 & 0,01 & 0,0001 & 0,33 & 0,02 & 0,004 & 0,00002 & 0,2 \\
\hline
\end{tabular}


Continuación Tabla 4.

\begin{tabular}{|c|c|c|c|c|c|c|c|c|}
\hline \multirow{2}{*}{ Variable } & \multicolumn{4}{|c|}{ Población de referencia } & \multicolumn{4}{|c|}{ Población de no referencia } \\
\hline & Media & Desviación & Varianza & Coeficiente & Promedio & Desviación & Varianza & Coeficiente \\
\hline \multicolumn{9}{|c|}{ Etapa 4 (12 meses de edad) } \\
\hline$N\left(g ~ k g^{-1}\right)$ & 13,30 & 1,52 & 2,30 & 0,11 & 19,51 & 1,36 & 1,84 & 0,069 \\
\hline$P\left(g_{k g}^{-1}\right)$ & 1,04 & 0,06 & 0,00 & 0,04 & 0,94 & 0,05 & 0,00 & 0,05 \\
\hline $\mathrm{K}\left(\mathrm{g} \mathrm{kg}^{-1}\right)$ & 56,48 & 4,28 & 18,35 & 0,07 & 40,78 & 13,02 & 169,56 & 0,31 \\
\hline Ca $\left(\mathrm{g} \mathrm{kg}^{-1}\right)$ & 2,25 & 0,27 & 0,07 & 0,11 & 2,91 & 1,56 & 2,43 & 0,53 \\
\hline $\operatorname{Mg}\left(\mathrm{kg}^{-1}\right)$ & 1,85 & 0,19 & 0,03 & 0,09 & 2,33 & 1,16 & 1,35 & 0,49 \\
\hline $\mathrm{Na}\left(\mathrm{g} \mathrm{kg}^{-1}\right)$ & 0,11 & 0,02 & 0,001 & 0,18 & 0,09 & 0,01 & 0,00 & 0,11 \\
\hline $\mathrm{Cu}\left(\mathrm{g} \mathrm{kg}^{-1}\right)$ & 0,02 & 0,01 & 0,0001 & 0,43 & 0,02 & 0,00 & 0,00 & 0,2 \\
\hline $\mathrm{Zn}\left(\mathrm{g} \mathrm{kg}^{-1}\right)$ & 0,05 & 0,02 & 0,0003 & 0,2 & 0,04 & 0,01 & 0,00 & 0,17 \\
\hline $\operatorname{Mn}\left(\mathrm{g} \mathrm{kg}^{-1}\right)$ & 0,32 & 0,05 & 0,003 & 0,156 & 0,51 & 0,11 & 0,01 & 0,21 \\
\hline $\mathrm{Fe}\left(\mathrm{g} \mathrm{kg}^{-1}\right)$ & 0,0001 & 0,00002 & 0,000 & & 0,0001 & 0,00 & 0,00 & \\
\hline$S\left(g_{k g}^{-1}\right)$ & 1,91 & 0,12 & 0,01 & 0,05 & 1,88 & 0,15 & 0,02 & 0,07 \\
\hline$B\left(g_{k g}^{-1}\right)$ & 0,03 & 0,003 & 0,00001 & 0,09 & 0,03 & 0,00 & 0,00 & 0,03 \\
\hline
\end{tabular}

incluso la población de no referencia presenta mayores valores de absorción en la primera etapa comparada con la población de referencia.

El comportamiento de los demás elementos se puede clasificar en tres grupos, Ca-Mg-Mn-Fe presentaron mayor absorción en las poblaciones de no referencia y tuvieron picos de mayor absorción en diferentes etapas de desarrollo del cultivo en ambas poblaciones.

Por otro lado, Zn, B y Na presentan mayor absorción en las poblaciones de referencia con respecto a las poblaciones de no referencia, el $\mathrm{Zn}$ tuvo un comportamiento de absorción decreciente, las primeras etapas tuvieron mayor demanda y al final del ciclo la demanda disminuyó, el B presentó mayores valores de absorción en el segundo ciclo del cultivo mientras el $\mathrm{Na}$ va aumenta su requerimiento.

El Cu y el S presentaron comportamientos de absorción y concentraciones similares en las poblaciones de referencia y de no referencia.

\section{Obtención de la norma o relaciones ideales}

Las relaciones que se convirtieron en normas fueron seleccionadas con base a las diferencias significativas entre las varianzas (Franco, 2007) para cada una de las etapas de desarrollo del cultivo. Del análisis total de las muestras foliares por etapas se eligió un número total de 66 relaciones de las 132, esto concuerda con el estudio realizado en caucho por Chacón et al. (2012) en donde seleccionó de las $132 \mathrm{RP}$ un total de $66 \mathrm{y}$ que además considero como importantes las relaciones N/P, N/K y N/Ca.

De las 66 relaciones ideales escogidas para cada una de las etapas de crecimiento del cultivo, se observa que 22 tiene una varianza alta, las relaciones $\mathrm{Mg} / \mathrm{S}$ y Na/Cu presentaron varianzas demasiado negativas, mientras 42 mostraron menor variabilidad, entre los que se destaca las relaciones de $\mathrm{K}$ con la mayoría de los elementos.

\section{Obtención de los índices DRIS}

De los análisis foliares de cada una las etapas se realizaron el diagnóstico nutricional junto con las normas DRIS obtenidas para cada una de las etapas. Hay que tener en cuenta que esta metodología dice que la interpretación se valida con la estimación de los índices para cada par de nutrientes, tanto de la muestra foliares tomada en campo como de las relaciones ideales DRIS correspondientemente (Chacón et al, 2012). En la tabla 5 se muestran los índices DRIS para cada elemento en todas etapas de desarrollo del cultivo.

Se manifiesta que los coeficientes de variación no están en porcentajes, por lo tanto, hay valores muy 
Tabla 5. Índices DRIS de interpretación de los diferentes nutrientes para las cuatro etapas de desarrollo en el cultivo de piña 'Oro Miel'.

\begin{tabular}{|l|r|r|r|r|}
\hline \multicolumn{5}{|c|}{ Índices DRIS } \\
\hline Nutriente & \multicolumn{1}{|c|}{ Etapa 1 } & \multicolumn{1}{|c|}{ Etapa 2 } & \multicolumn{1}{c|}{ Etapa 3 } & \multicolumn{1}{c|}{ Etapa 4 } \\
\hline N & 176,83 & 4,30 & $-2,86$ & $-12,87$ \\
\hline P & 278,09 & 191,55 & 97,87 & 285,16 \\
\hline K & $-20,11$ & $-3,97$ & 0,41 & 9,93 \\
\hline Ca & $-70,92$ & $-635,57$ & $-160,49$ & $-101,86$ \\
\hline Mg & 20,53 & $-46,51$ & 1640,89 & $-48,4$ \\
\hline Na & 6183,73 & $-1430,65$ & 4344,49 & 126,82 \\
\hline Cu & 45,44 & 76909,78 & 7861,65 & 14417,24 \\
\hline Zn & 894,71 & $-39449,85$ & $-2665,65$ & $-6676,75$ \\
\hline Mn & $-76239,88$ & $-653,5$ & -590 & $-558,86$ \\
\hline Fe & 75743,95 & & & \\
\hline S & $-99,02$ & 323,30 & $-1504,29$ & 73,10 \\
\hline B & $-1084,72$ & $-35116,55$ & $-9022,9$ & $-7582,99$ \\
\hline IBN ${ }^{1}$ & 160857,95 & 154765,53 & 27891,5 & 29893,98 \\
\hline IBNm ${ }^{2}$ & 13404,82 & 12897,12 & 2324,29 & 2491,16 \\
\hline
\end{tabular}

IIBN: índice de balance nutricional.

${ }^{2} \mid \mathrm{BNm}$ : índice de balance nutricional medio.

pequeños comparados con valores grandes, es por ese motivo que algunos valores de índices DRIS son tan altos. Además de ello que los valores del análisis foliar todos se encontraban unidades de $\mathrm{g} \mathrm{kg}^{-1}$, por lo tanto, había valores muy pequeños en gramos, principalmente en los micronutrientes, y los elementos mayores no se encontraban en porcentaje. Esto último se hizo con el fin de estandarizar los valores de los análisis foliares.

\section{Interpretación de los índices DRIS}

De acuerdo con la metodología reportado por Beaufils (1973) y clasificada por Wadt (1996), los resultados de los índices DRIS para los primeros 3 meses de desarrollo del cultivo de piña muestran que el elemento que presentó deficiencia fue el $\mathrm{Mn}$ y que los nutrientes $\mathrm{N}, \mathrm{P}, \mathrm{Mg}, \mathrm{Na}, \mathrm{Cu}$ y $\mathrm{Zn}$ estaban en equilibrio. Por otra parte, el elemento Fe, fue el que mostró excesos, pues el índice era superior IBNm.

Los nutrientes que presentaron mayor deficiencia, para la primera etapa desarrollo, se encuentran en el siguiente orden: $\mathrm{Mn}>\mathrm{B}>\mathrm{S}>\mathrm{Ca}>\mathrm{K}>\mathrm{Mg}>\mathrm{Cu}>\mathrm{N}>\mathrm{P}>\mathrm{Zn}>\mathrm{Na}>\mathrm{Fe}$.
El Fe fue uno de los elementos que presentó mayor variabilidad en las dos poblaciones, pues se vio que en la primera etapa de desarrollo se encontraba en cantidad probablemente altas, pero que cuando fue avanzada la edad del cultivo este disminuyó considerablemente.

Ya para la segunda etapa de desarrollo del cultivo, el $\mathrm{B}$ y el Zn fueron los elementos más limitantes para el desarrollo vegetal por déficit. La deficiencia de B puede ser el causando de grietas en el fruto (Malézieux y Bartholomew, 2003), pero que no fueron observados en el cultivo. Los nutrientes N, P y S se encontraban equilibrados. Y el $\mathrm{Cu}$ fue para esta etapa de desarrollo el más excesivo.

Los nutrientes que presentaron mayor deficiencia para la segunda etapa de desarrollo del cultivo se encontraban en el siguiente orden de acuerdo con los índices DRIS: $\mathrm{Zn}>\mathrm{B}>\mathrm{Na}>\mathrm{Ca}>\mathrm{Mn}>\mathrm{Mg}>\mathrm{K}>\mathrm{N}>\mathrm{P}>\mathrm{S}>\mathrm{Cu}$.

En la tercera etapa de desarrollo del cultivo se pudo observar que los elementos B y $S$ se encontraban en un estado limitante del desarrollo vegetal por deficiencia por lo contrario el Cu seguía presentando excesos en la planta, como también el $\mathrm{Na}$. El P fue uno de los elementos que permaneció equilibrado, siguiendo con la tendencia de la primera etapa.

El orden de los nutrientes que presentaron mayor deficiencia para la tercera etapa de desarrollo del cultivo de piña fueron los siguientes: $\mathrm{B}>\mathrm{Zn}>\mathrm{S}>\mathrm{Mn}>\mathrm{Ca}>\mathrm{N}>\mathrm{K}>\mathrm{P}>\mathrm{Mg}>\mathrm{Na}>\mathrm{Cu}$.

Por último, B y $\mathrm{Zn}$ siguieron presentando deficiencias, el $\mathrm{Cu}$ fue el nutriente más limitante por excesos en esta etapa, mientras el $\mathrm{P}$ permaneció en estado equilibrado durante toda la etapa del cultivo, teniendo en cuenta que el $\mathrm{P}$ contribuye al crecimiento en todas las partes de esta especie frutal (Vásquez et al., 2012). Los demás elementos no presentaron un valor acorde a los establecidos en el parámetro de interpretación DRIS.

El orden de los nutrientes que presentaron mayor deficiencia para la última etapa de muestreo fue el siguiente: $\mathrm{B}>\mathrm{Zn}>\mathrm{Mn}>\mathrm{Ca}>\mathrm{Mg}>\mathrm{N}>\mathrm{K}>\mathrm{S}>\mathrm{Na}>\mathrm{P}>\mathrm{Cu}$.

El comportamiento de los nutrientes en cada una de las etapas concuerda con lo reportado en el cultivo de palma (Herrera, 2015), donde los elementos menores se encuentran en menor limitación y los elementos mayores en mayor limitación, mientras en un estudio 
realizado en nogal, Medina (2004) reporta que el Mn es el elemento que se encuentra en mayor limitación.

\section{CONCLUSIONES}

El P, N y K no presentaron limitaciones por excesos ni por defecto lo que puede traducirse en que la planta asimiló las aplicaciones de fertilizaciones de manera eficiente.

Las dosis de los tratamientos propuestos lograron suplir los requerimientos del cultivo, sin embargo, es necesario tener en cuenta las cantidades aplicadas para que mantengan la relación entre los nutrientes y no haya desbalances nutricionales sobre todo con los elementos menores.

\section{AGRADECIMIENTOS}

Esta investigación se llevó a cabo gracias a la financiación de la Alcaldía del Municipio de Santander de Quilichao y la Universidad Nacional de Colombia en el marco del proyecto "Determinación de los requerimientos nutricionales de la piña variedad MD-2 en suelos ácidos del Municipio de Santander de Quilichao". Los autores agradecen especialmente a la Asociación Frupasa de la vereda Alegrías, al profesor Héctor Fabio Ramos y al grupo de tesistas.

Conflicto de intereses: el manuscrito fue preparado y revisado con la participación de los autores, quienes declaran no tener algún conflicto de interés que coloquen en riesgo la validez de los resultados aquí presentados.

\section{REFERENCIAS BIBLIOGRÁFICAS}

Angeles, D., M. Sumner y N. Barbour. 1990. Preliminary nitrogen, phosphorus and potassium DRIS norms for pineapple. HortScience 25, 652-655.

Baldock, J.O. y E.E. Schulte. 1996. Plant analysis with standardized scores combines DRIS and sufficiency range approaches for corn. Agron. J. 88, 448-456. Doi: 10.2134/agronj1996.00021962008800030015x

Beaufils, E. 1973. Diagnosis and Recommendation Integrated System (DRIS): a general scheme for experimentation and calibration based on principles developed from research in plant nutrition. Soil Science Bulletin No. 1. Department of Soil Science and Agrometeorology University of Natal, Pietermaritzburg, Sudáfrica.
Beaufils, E. y M. Sumner. 1976. Application of the DRIS approach for calibrating soil, plant yield and quality factors of sugarcane. Sugar Tech. Assoc. 50, 118-124.

Bell, P., W. Hallmark, W. Sabbe y D. Dombeck. 1995. Diagnosing nutrient deficiencies in soybean, using M-DRIS and critical nutrient level procedures. Agron. J. 87, 859-865. Doi: 10.2134/agronj1995.00021962008 $700050013 x$

Cerda, A., M. Nieves y V. Martínez. 1995. An evaluation of mineral analysis of Verna lemons by DRIS. Com. Soil Sci. Plant Anal. 26, 1697-1707. Doi: 10.1080/00103629509369402

Chacón, E. 2012. Obtención de la norma de diagnóstico y recomendación integral (DRIS) para el cultivo de caucho (Hevea brasiliensis) en la Altillanura Colombiana. Tesis de doctorado. Facultad de Agronomía, Universidad Nacional de Colombia, Bogotá, Colombia.

CIAT (Centro Internacional de Agricultura Tropical). 1993. Manual de análisis de suelos y tejido vegetal. Guía Teórica y práctica de metodologías. Cali, Colombia.

Combatt, E., A. Jarma y A. Cleves 2008. Efecto del encalamiento de suelos sulfatados ácidos en Córdoba (Colombia) sobre el fruto de piña (Ananas comosus (L.) Merr.). Rev. Colomb. Cienc. Hortic. 2(2), 173-179. Doi: $10.17584 /$ rcch.2008v2i2.1185

Da Costa, A. y L. Prezotti. 1997. Padrao de referencia para uso do DRIS na avaliçao nutricional do café arábica. Inf. Agr. 80, 9-10.

Elwali, A. y G. Gascho. 1984. Soil testing, foliar analysis, and DRIS as guides for sugarcane fertilization. Agron. J. 76, 466-470. Doi: 10.2134/agronj1984.00021962007 $600030024 x$

Franco, H. 2007. Determinación de normas de diagnóstico nutricional para el cultivo de rosa de corte en Colombia. Tesis de maestría. Facultad de Agronomía, Universidad Nacional de Colombia. Bogotá, Colombia.

García, M. y H. Serrano. 2005. La piña, Ananas comosus (L.) Merr. (Bromeliaceae), algo más que un fruto dulce y jugoso. ContactoS, 56, 55-61.

García, S. 2000. Establecimiento de normas DRIS, diagnóstico nutricional y calibración de las normas obtenidas para el cultivo de papa (Solanum tuberosum L.) en Coahuila y Nuevo León. Tesis de doctorado. Facultad de Agronomía, Universidad Autónoma Nuevo León, Marín, México.

Hassan, A., Z. Othman y J. Siriphanich. 2011. Pineapple (Ananas comosus L. Merr.). pp. 194-218. En: Yahia, E.M. (ed.). Postharvest biology and technology of tropical and subtropical fruits. Vol. 4: Mangosteen to white sapote. Woodhead Publishing, Oxford, UK. Doi: 10.1533/9780857092618.194

Herrera, G. 2015. Obtención del sistema integrado de diagnóstico y recomendación integral (DRIS) en el cultivo 
de palma de aceite (Elaeis guineensis Jacq.). Tesis de maestría. Facultad de Agronomía, Universidad Nacional de Colombia, Bogotá, Colombia.

Ideam (Instituto de Hidrología, Metereología y Estudios Ambientales). 2016. Informe Sistema de Información para Santander de Quilichao, Cauca. En: www.ideam. gov.co/; consulta: octubre de 2017.

León, J. 2000. Botánica de los cultivos tropicales. 3a ed. Agroamérica Instituto Interamericano para la Agricultura, San José, Costa Rica. pp. 456-460.

Letzsch, W. y M. Sumner. 1984. Effect of population size and yield level in selection of diagnosis and recommendation integrated system (DRIS) norms. Com. Soil Sci. 15, 997-1006. Doi: 10.1080/00103628409367537

Malézieux, E. y D. Bartholomew. 2003. Plant nutrition. pp. 143-165. En: Bartholomew, D.P., R.E. Paull y K.G. Rohrbach (eds.). The pineapple, botany, production and uses. Cabi Publishing, Wallingford, UK. Doi: 10.1079/9780851995038.0143

Marca-Huamancha, C., R. Borjas-Ventura, D. Rebaza-Fernández, S. Bello-Amez y A. Julca-Otiniano. 2018. Efecto de la fertilización mineral y de un fertilizante biológico en piña [Ananas comosus (L.) Merr.] en el cultivar MD2. Rev. Colomb. Cienc. Hortic. 12(1), 59-68. Doi: $10.17584 /$ rcch.2018v12i1.7901

Marschner, P. (ed.). 2011. Marschner's mineral nutrition of higher plants. $3^{\text {rd }}$ ed. Academic Press; Elservier, Amsterdam, Holanda. Doi: 10.1016/C2009-0-63043-9

Medina-Morales, M.C. 2004. Normas DRIS preliminares para nogal pecanero. Terra Lationoam. 22(4), 445-450.

Ministerio de Agricultura y Desarrollo Rural. 2016. Cadena de piña. Indicadores e instrumentos. Bogotá, Colombia.

Mourão-Filho, F. 2004. Concepts and applications on nutritional diagnosis in fruit crops. Sci. Agri. 61, 550-560. Doi: 10.1590/S0103-90162004000500015

Rebolledo M., A., A.L. del Ángel P., L. Rebolledo M., A.E. Bacerril R. y D. Uriza-Ávila. 2006. Rendimiento y calidad de fruto de cultivares de piña en densidades de plantación. Rev. Fitotec. Mex. 19(1), 55-62.

Rodríguez, O. y V. Rodríguez. 2000. Desarrollo, determinación e interpretación de normas DRIS para el diagnóstico nutricional en plantas. Rev. Fac. Agron. (LUZ) 17, 449-470.

Sánchez G., A.A., J. Giralí C., L. Zérega, O.A. Rodríguez R., S.A. Oliveira y V.J. Rodríguez P. 2009. Normas preliminares DRIS desarrolladas para caña de azúcar a partir de un bajo número de muestras. Pesq. Agropec. Bras. 44(12), 1700-1706. Doi: 10.1590/ S0100-204X2009001200020

Sema, A., C. Maiti, A. Singh y A. Bendangsengla. 2010. Dris nutrient norms for pineapple on alfisols of India. J. Plant Nutr. 33, 1384-1399. Doi: 10.1080/01904167.2010.484286

Sharma, J., S. Shikhamany, R. Singh y H. Raghupathi. 2005. Diagnosis of nutrient imbalance in Thompson Seedless grape grafted on Dog Ridge rootstock by DRIS. Com. Soil Sci. Plant 36, 2823-2838. Doi: 10.1080/00103620500305991

Sumner, M. 1977. Use of the DRIS System in foliar diagnosis of crops at high yield levels. Com. Soil Sci. Plant. 8(3), 251-288. Doi: 10.1080/00103627709366718

Urricariet, S., R. Lavado y L. Martín. 2004. Corn response to fertilization and SR, DRIS, and PASS interpretation of leaf and grain analysis. Com. Soil Sci. Plant 35, 413 425. Doi: 10.1081/CSS-120029722

Wadt, P. 1996. Os métodos da chance matemática e do Sistema, Integrado de Diagnose e Recomendação (DRIS) na avaliação nutricional de plantios de eucalipto. Tesis de doctorado. Universidade Federal de Viçosa, Viçosa, Brasil.

Vásquez, H.D., R. Saavedra O. y S.H. Saavedra S. 2012. Piña (Ananas comosus L. Merr). pp. 776-801. En. Fischer, G. (ed.). Manual para el cultivo de frutales en el trópico. Produmedios, Bogotá, Colombia. 